\title{
On the correlation of the truncated Liouville function
}

\author{
by
}

\author{
HÉdi Daboussi (Amiens) and András SÁRKözy (Budapest)
}

1. Introduction. $\mathbb{Z}$ and $\mathbb{N}$ denote the set of the integers and positive integers, respectively. $\omega(n)$ denotes the number of prime factors of $n$, while $\Omega(n)$ is the number of prime factors of $n$ counted with multiplicity. $\lambda(n)$ denotes the Liouville function: $\lambda(n)=(-1)^{\Omega(n)}$. If $f(n)$ is a multiplicative function and $y>0$, then $f_{y}(n)$ will denote the multiplicative function defined by

$$
f_{y}\left(p^{\alpha}\right)=\left\{\begin{array}{ll}
f\left(p^{\alpha}\right) & \text { for } p \leq y, \\
1 & \text { for } p>y,
\end{array} \quad \alpha \in \mathbb{N} .\right.
$$

In particular, $\lambda_{y}(n)$ denotes the "truncated" Liouville function which is completely multiplicative and

$$
\lambda_{y}(p)= \begin{cases}-1(=\lambda(p)) & \text { for } p \leq y, \\ +1 & \text { for } p>y .\end{cases}
$$

If $f(n)$ is an arithmetic function and $x>0$, then we write

$$
H(f, x)=\sum_{n \leq x} f(n) f(n+1) .
$$

The estimate of the correlation of the Liouville function is a hopelessly difficult task and as Hildebrand [6] writes: "(...) one would naturally expect that the above sum" (the sum $H(\lambda, x)$ ) "is of order $o(x)$ when $x \rightarrow \infty$, but even the much weaker relation

$$
\liminf _{x \rightarrow \infty} \frac{H(\lambda, x)}{x}<1
$$

is not known and seems to be beyond reach of the present methods". Thus Cassaigne et al. in [3] proposed to study the "truncated" Liouville function

2000 Mathematics Subject Classification: Primary 11K45.

Research of the second author is partially supported by Hungarian National Foundation for Scientific Research, Grant no. T029759. This paper was written while the second author was visiting the Université de Picardie, Amiens. 
instead, i.e., to estimate $\left|H\left(\lambda_{y}, x\right)\right|$. They showed that for $x \geq 2$ and

$$
2 \leq y \leq(\log x)^{2} /(\log \log x)^{2}
$$

we have

$$
\left|H\left(\lambda_{y}, x\right)\right|<c_{1} \frac{x}{(\log y)^{4}} .
$$

$\left(c_{1}, c_{2}, \ldots\right.$ will denote positive absolute constants.) Thus for $y \rightarrow \infty$ and

$$
y \leq(\log x)^{2} /(\log \log x)^{2}
$$

we have

$$
H\left(\lambda_{y}, x\right)=o(x) .
$$

In [4] we extended the problem to completely multiplicative arithmetic functions $f(n)$ with

$$
f(n) \in\{-1,+1\} \quad \text { for all } n \in \mathbb{N},
$$

and we considerably improved on the estimates given in [3]. In particular, we proved that (1.1) holds under the condition

$$
(2 \leq) y \leq x^{1 /(501 \log \log x)}
$$

which is much weaker than the one in (1.2). Moreover, we wrote: "We think that (...) even the upper bound

$$
y=x^{o(1)}
$$

can be achieved. However, there are certain technical difficulties in doing this, we hope to return to this problem in a subsequent paper". Indeed, in this paper our goal is to prove (1.3) under condition (1.5); this result seems to be the limit of our knowledge in this direction at the present. The crucial step in improving the bound in (1.4) to the one in (1.5) is to replace Brun's "simple" or "pure" sieve in the argument given in [4] by the complete, strongest form of Brun's sieve. To force out the applicability of the latter, we will need two new lemmas (Lemmas 3 and 6 below), and later the introduction of the set $\mathcal{N}_{3}$ and the estimate of the error term $R_{3}$ will also serve this purpose. There will also be some minor changes, but a considerable part of the technical details will remain unchanged; in these cases, we will omit the details here and instead we will refer to [4].

We will prove:

THEOREM. If $f(n)$ is a multiplicative arithmetic function with

$$
|f(n)| \leq 1 \quad \text { for all } n \in \mathbb{N}
$$

and $2 \leq y \leq x$, then, writing

$$
u=\frac{\log x}{\log y},
$$


we have

$$
\left|H(f, x)-x \prod_{p \leq y} \delta(p)\right| \ll x\left((\log y)^{-9}+\exp \left(-\frac{4}{8}\right)+\sum_{\substack{y<p \leq x \\ f(p) \neq 1}} \frac{1}{p}\right)
$$

where $\delta(p)$ is defined by

$$
\delta(p)= \begin{cases}\sum_{\alpha=1}^{\infty} \frac{f\left(2^{\alpha}\right)}{2^{\alpha}} & \text { for } p=2, \\ \left(1-\frac{2}{p}\right)\left(1+2 \frac{p-1}{p-2} \sum_{\beta=1}^{\infty} \frac{f\left(p^{\beta}\right)}{p^{\beta}}\right) & \text { for } p>2 .\end{cases}
$$

If $f(n)$ is completely multiplicative, then

$$
\delta(p)=\frac{p+f(p)-2}{p-f(p)} .
$$

Applying this theorem with $f_{y}(n)$ in place of $f(n)$, clearly we obtain:

COROLlary 1. If $f(n)$ is a multiplicative arithmetic function satisfying (1.6), $2 \leq y \leq x$, and $u$ is defined by (1.7), then

$$
\left|H\left(f_{y}, x\right)-x \prod_{p \leq y} \delta(p)\right| \ll x\left((\log y)^{-9}+\exp \left(-\frac{u}{8}\right)\right)
$$

where $\delta(p)$ is defined by (1.9), and if $f(n)$ is completely multiplicative, then the latter can be replaced by (1.10).

In particular, if $f(n)=\lambda(n)$, then in (1.10) we have

$$
\delta(3)=\frac{3+\lambda(3)-2}{3-\lambda(3)}=0 .
$$

Thus it follows from Corollary 1 that

Corollary 2. If $3 \leq y \leq x$ and $u$ is defined by (1.7) then

$$
\left|H\left(\lambda_{y}, x\right)\right| \ll x\left((\log y)^{-9}+\exp \left(-\frac{u}{8}\right)\right) .
$$

Thus, indeed, (1.3) holds for $y$ satisfying $y \rightarrow \infty$ and (1.5).

2. Lemmas. Let $Q_{y}$ denote the set of the positive integers all of whose prime factors are $\leq y$ (including $1 \in Q_{y}$ ), and write

$$
P_{y}=\prod_{p \leq y} p, \quad B_{y}=\sum_{p \leq y} \frac{1}{p} .
$$

Define the completely multiplicative arithmetic functions $d(n), m(n)$ by

$$
n=d(n) m(n), \quad d(n), m(n) \in \mathbb{N}, d(n) \in Q_{y},\left(m(n), P_{y}\right)=1 .
$$


(In other words, $d(n)$ collects the prime factors of $n$ not exceeding $y$, while $m(n)$ is composed of the prime factors $>y$.) Then we clearly have

$$
f_{y}(n)=f(d(n)) .
$$

$\psi(x, y)$ denotes the number of integers $\leq x$ all of whose prime factors are $\leq y$. We will need several lemmas.

Lemma 1. Let $2 \leq y \leq x, k \in \mathbb{N}, a_{1}, \ldots, a_{k} \in \mathbb{N}, b_{1}, \ldots, b_{k} \in \mathbb{Z},\left(a_{i}, a_{j}\right)$ $=1$ for $1 \leq i<j \leq k$, and $\left(a_{i}, b_{i}\right)=1$ for $i=1, \ldots, k$. Set

$$
v=\frac{\log x}{\log y}, \quad E=\prod_{i=1}^{k} a_{i} \prod_{1 \leq i<j \leq k}\left(a_{i} b_{j}-a_{j} b_{i}\right), \quad F(n)=\prod_{i=1}^{k}\left(a_{i} n+b_{i}\right),
$$

and denote the number of solutions of the congruence $F(x) \equiv 0(\bmod m)$ by $\varrho(m)$. Then for $x \rightarrow \infty$ we have

$$
\begin{aligned}
& \left|\left\{n: n \leq x,\left(F(n), P_{y}\right)=1\right\}\right| \\
& =x \prod_{\substack{p \mid E \\
p \leq y}}\left(1-\frac{\varrho(p)}{p}\right) \prod_{\substack{p \nmid E \\
p \leq y}}\left(1-\frac{k}{p}\right) \\
& \quad \times(1+O(\exp (-v(\log v-\log \log 3 v-\log k-2)))+O(\exp (-\sqrt{\log x}))) .
\end{aligned}
$$

Proof. This is a special case of the "fundamental lemma" type Brun sieve result Theorem 2.5 in [5].

Lemma 2. If $2 \leq y \leq x$, then

$$
\left|\left\{n: n \leq x,\left(n, P_{y}\right)=1\right\}\right| \ll \frac{x}{\log y} .
$$

Proof. This is a special case of Lemma 2 in [4] and, indeed, it follows from Lemma 1 above (with $F(n)=n$ ).

Lemma 3. Assume that $h(n)$ is a multiplicative function such that

$$
h(p)=h\left(p^{2}\right)=\ldots=h\left(p^{\alpha}\right)=\ldots \quad \text { for all } p,
$$

and there are numbers $K>0, L \geq 0$ with

$$
|h(p)-K| \leq L / p \quad \text { for all } p .
$$

Then for all $2 \leq y \leq z$ we have

$$
\sum_{\substack{z \leq n \\ n \in Q_{y}}} \frac{h(n)}{n} \ll(\log y)^{K} \exp \left(-\frac{\log z}{\log y}\right)
$$

where the implicit constant depends on $K$ and $L$ only. 
Proof. For all $\eta>0$ we have

$$
\sum_{\substack{z \leq n \\ n \in Q_{y}}} \frac{h(n)}{n} \leq \sum_{\substack{z \leq n \\ n \in Q_{y}}} \frac{h(n)}{n}\left(\frac{n}{z}\right)^{\eta}=\frac{1}{z^{\eta}} \sum_{\substack{z \leq n \\ n \in Q_{y}}} \frac{h(n)}{n^{1-\eta}} .
$$

Choosing $\eta=1 / \log y$ here, we obtain

$$
\begin{aligned}
\sum_{\substack{z \leq n \\
n \in Q_{y}}} \frac{h(n)}{n} & \leq \exp \left(-\frac{\log z}{\log y}\right) \sum_{n \in Q_{y}} \frac{h(n)}{n^{1-\eta}} \\
& =\exp \left(-\frac{\log z}{\log y}\right) \prod_{p \leq y}\left(1+h(p) \sum_{\alpha=1}^{\infty} \frac{1}{p^{\alpha(1-\eta)}}\right) \\
& =\exp \left(-\frac{\log z}{\log y}\right) \prod_{p \leq y}\left(1+\frac{h(p)}{p^{1-\eta}-1}\right) .
\end{aligned}
$$

Here we have

$$
\begin{aligned}
\prod_{p \leq y}\left(1+\frac{h(p)}{p^{1-\eta}-1}\right) & =\exp \left(\sum_{p \leq y} \frac{h(p)}{p^{1-\eta}}+O(1)\right) \\
& =\exp \left(\sum_{p \leq y} \frac{h(p)}{p} \exp (\eta \log p)+O(1)\right) \\
& =\exp \left(\sum_{p \leq y} \frac{h(p)}{p}(1+\eta \log p)+O(1)\right) \\
& =\exp \left(\sum_{p \leq y} \frac{h(p)}{p}+\eta \sum_{p \leq y} \frac{h(p) \log p}{p}+O(1)\right) \\
& =\exp (K \log \log y+\eta K \log y+O(1)) \ll(\log y)^{K} .
\end{aligned}
$$

Now (2.5) follows from (2.6) and (2.7).

Lemma 4. For all $2 \leq y \leq z$ we have

$$
\sum_{\substack{z \leq n \\ n \in Q_{y}}} \frac{1}{n} \ll(\log y) \exp \left(-\frac{\log z}{\log y}\right) .
$$

Proof. This is Lemma 3 in [4] and, indeed, it follows from Lemma 3 above with $h(n) \equiv 1, K=1, L=0$.

Lemma 5. If $(\log x)^{2} \leq y \leq x$ and $u$ is defined by (1.7), then

$$
\psi(x, y) \ll x \exp (-u \log u) .
$$

Proof. This is Lemma 4 in [4] and, indeed, this follows from de Bruijn's classical estimate (see Theorem 2 in Part II of [1]; see also [2]). 
Lemma 6. Defining $d(n)$ by (2.1) and u by (1.7), we have

$$
\sum_{\substack{n \leq x \\ d(n)>x^{1 / 8}}} 1 \ll x \exp \left(-\frac{u}{8}\right) .
$$

Proof. We have

$$
\sum_{\substack{n \leq x \\ d(n)>x^{1 / 8}}} 1=\sum_{\substack{n \leq x \\ x^{1 / 8}<d(n) \leq x / y}} 1+\sum_{\substack{n \leq x \\ x / y<d(n)}} 1=K_{1}+K_{2},
$$

say. If $n$ is counted in the first term, then it is of the form $n=d m$ with $x^{1 / 8}<d \leq x / y, d \in Q_{y},\left(m, P_{y}\right)=1$ so that, using first Lemma 2 and then Lemma 4 , we get

$$
\begin{aligned}
K_{1} & \leq \sum_{\substack{x^{1 / 8}<d \leq x / y \\
d \in Q_{y}}} \sum_{\substack{m \leq x / d \\
\left(m, P_{y}\right)=1}} 1 \\
& \ll \sum_{\substack{x^{1 / 8}<d \leq x / y \\
d \in Q_{y}}} \frac{x}{d \log y} \leq \frac{x}{\log y} \sum_{\substack{n^{1 / 8}<d \\
d \in Q_{y}}} \frac{1}{d} \\
& \ll \frac{x}{\log y}(\log y) \exp \left(-\frac{\log x^{1 / 8}}{\log y}\right)=x \exp \left(-\frac{u}{8}\right) .
\end{aligned}
$$

If $n$ is counted in the second term in (2.9), then in $n=d(n) m \leq x$, $\left(m, P_{y}\right)=1$ we have

$$
m \leq \frac{x}{d(n)}<y
$$

so that we must have $m=1$, thus

$$
K_{2}=\sum_{\substack{x / y<d \leq x \\ d \in Q_{y}}} 1 \leq \sum_{\substack{d \leq x \\ s \in Q_{y}}} 1=\psi(x, y) .
$$

If $(\log x)^{2} \leq y$, then by Lemma 5 it follows that

$$
K_{2} \ll \exp (-u \log u) \quad\left(\text { for }(\log x)^{2} \leq y\right) .
$$

If $y<(\log x)^{2}$, then again by Lemma 5 we have

$$
\begin{aligned}
K_{2} & \leq \psi(x, y) \leq \psi\left(x,(\log x)^{2}\right) \\
& \ll x \exp \left(-\frac{\log x}{\log (\log x)^{2}} \log \frac{\log x}{\log (\log x)^{2}}\right) \\
& =x \exp \left(-\left(\frac{1}{2}+o(1)\right) \log x\right)
\end{aligned}
$$




$$
\begin{aligned}
& =x^{1 / 2+o(1)} \ll x \exp \left(-\frac{1}{8} \cdot \frac{\log x}{\log 2}\right) \\
& \leq x \exp \left(-\frac{1}{8} u\right) \quad\left(\text { for } 2 \leq y<(\log x)^{2}\right) .
\end{aligned}
$$

Now (2.8) follows from (2.9)-(2.12).

3. Completion of the proof of the Theorem. Clearly we have $f(n)=f_{y}(n)$ unless there is a prime $p$ with $p>y, f(p) \neq 1, p \mid n$.

Thus it follows in the same way as in (4.1) of [4] that

$$
\left|\sum_{n \leq x} f(n) f(n+1)-\sum_{n \leq x} f_{y}(n) f_{y}(n+1)\right| \leq 6 x \sum_{\substack{y<p \leq x \\ f(p) \neq 1}} \frac{1}{p}+2 .
$$

Thus it suffices to estimate $H\left(f_{y}, x\right)=\sum_{n \leq x} f_{y}(n) f_{y}(n+1)$.

Set

$$
\phi=60 B_{y}=60 \sum_{p \leq y} \frac{1}{p}
$$

and define $\mathcal{N}_{0}, \mathcal{N}_{1}, \mathcal{N}_{2}$ and $\mathcal{N}_{3}$ by

$$
\begin{aligned}
& \mathcal{N}_{1}=\{n: n \leq x, \omega(d(n) d(n+1))>\phi\}, \\
& \mathcal{N}_{2}=\{n: n \leq x, \Omega(d(n) d(n+1))-\omega(d(n) d(n+1))>\phi\}, \\
& \mathcal{N}_{3}=\left\{n: n \leq x, d(n) d(n+1)>x^{1 / 4}\right\}
\end{aligned}
$$

and

$$
\mathcal{N}_{0}=\{n: n \leq x\} \backslash\left(\mathcal{N}_{1} \cup \mathcal{N}_{2} \cup \mathcal{N}_{3}\right)
$$

Then writing

$$
S=\sum_{n \in \mathcal{N}_{0}} f_{y}(n) f_{y}(n+1)
$$

by (1.6) we have

$$
\begin{aligned}
\left|\sum_{n \leq x} f_{y}(n) f_{y}(n+1)-S\right| & =\left|\sum_{n \in \mathcal{N}_{1} \cup \mathcal{N}_{2} \cup \mathcal{N}_{3}} f_{y}(n) f_{y}(n+1)\right| \\
& \leq \sum_{n \in \mathcal{N}_{1} \cup \mathcal{N}_{2} \cup \mathcal{N}_{3}}\left|f_{y}(n) f_{y}(n+1)\right| \\
& \leq \sum_{n \in \mathcal{N}_{1} \cup \mathcal{N}_{2} \cup \mathcal{N}_{3}} 1 \leq\left|\mathcal{N}_{1}\right|+\left|\mathcal{N}_{2}\right|+\left|\mathcal{N}_{3}\right|
\end{aligned}
$$

It remains to estimate $\left|\mathcal{N}_{1}\right|,\left|\mathcal{N}_{2}\right|,\left|\mathcal{N}_{3}\right|$ and $S$. 
$\left|\mathcal{N}_{1}\right|$ and $\left|\mathcal{N}_{2}\right|$ can be estimated in the same way as in [4], and first we obtain

$$
\left|\mathcal{N}_{1}\right| \leq 4 x \sum_{k>\phi / 2} \frac{1}{k !}\left(2 \sum_{p \leq y} \frac{1}{p}\right)^{k}
$$

whence, by Stirling's formula,

$$
\begin{aligned}
\left|\mathcal{N}_{1}\right| & \leq 4 x \sum_{k>\phi / 2}\left(\frac{3}{k}\right)^{k}\left(2 B_{y}\right)^{k} \\
& <4 x \sum_{k>\phi / 2}\left(\frac{12}{\phi} B_{y}\right)^{k}=4 x \sum_{k>\phi / 2} 5^{-k} \\
& \ll x 5^{-\phi / 2} \ll x(\log y)^{-30} .
\end{aligned}
$$

Next, as in [4], we obtain

$$
\left|\mathcal{N}_{2}\right| \ll \sum_{r^{2} \geq 2^{\phi / 2}} \frac{x}{r^{2}} \ll x 2^{-\phi / 4} \ll x(\log y)^{-9} .
$$

Moreover, we clearly have

$$
\begin{aligned}
\mathcal{N}_{3} \subset\left\{n: n \leq x, d(n)>x^{1 / 8}\right\} \\
\cup\left\{n: n+1 \leq x, d(n+1)>x^{1 / 8}\right\} \cup\{n: x<n+1 \leq x+1\}
\end{aligned}
$$

whence, by Lemma 6 ,

$$
\left|\mathcal{N}_{3}\right| \leq 2 \sum_{\substack{n \leq x \\ d(n)>x^{1 / 8}}} 1+1 \ll x \exp \left(-\frac{u}{8}\right) .
$$

Now consider the sum $S$ in (3.2), i.e. the summation over $n \in \mathcal{N}_{0}$. The definition of $\mathcal{N}_{0}$ can be rewritten as

$$
\begin{aligned}
\mathcal{N}_{0}= & \{n: n \leq x, \omega(d(n) d(n+1)) \leq \phi, \\
& \left.\Omega(d(n) d(n+1))-\omega(d(n) d(n+1)) \leq \phi, d(n) d(n+1) \leq x^{1 / 4}\right\} .
\end{aligned}
$$

In each term in (3.2), we write $n$ in the form (2.1), we use (2.2), and then we group the terms according to the values of $d(n)$ and $d(n+1)$ :

$$
\begin{aligned}
S & =\sum_{n \in \mathcal{N}_{0}} f(d(n)) f(d(n+1)) \\
& =\sum_{\left(d, d^{\prime}\right)} f(d) f\left(d^{\prime}\right)\left|\left\{n: n \in \mathcal{N}_{0}, d(n)=d, d(n+1)=d^{\prime}\right\}\right|
\end{aligned}
$$

where we sum over all pairs $\left(d, d^{\prime}\right)$ such that there is at least one $n$ with

$$
\begin{gathered}
n \in \mathcal{N}_{0}, \\
d(n)=d, \quad d(n+1)=d^{\prime} .
\end{gathered}
$$


If there is at least one $n$ with these properties, then we must have

$$
d=d(n) \in Q_{y}, \quad d^{\prime}=d(n+1) \in Q_{y},
$$

and by (3.7) and (3.9),

$$
\begin{aligned}
\omega\left(d d^{\prime}\right) & =\omega(d(n) d(n+1)) \leq \phi, \\
\Omega\left(d d^{\prime}\right)-\omega\left(d d^{\prime}\right) & =\Omega(d(n) d(n+1))-\omega(d(n) d(n+1)) \leq \phi, \\
d d^{\prime} & =d(n) d(n+1) \leq x^{1 / 4} .
\end{aligned}
$$

Moreover by (3.10) we have $d \mid n$ and $d^{\prime} \mid n+1$ whence

$$
\left(d, d^{\prime}\right)=1 \text {. }
$$

By (3.11)-(3.15), in the sum in (3.8) we may restrict ourselves to the set

$$
\begin{aligned}
\mathcal{E}=\left\{\left(d, d^{\prime}\right): d, d^{\prime} \in Q_{y}, \omega\left(d d^{\prime}\right) \leq \phi,\right. & \\
& \left.\Omega\left(d d^{\prime}\right)-\omega\left(d d^{\prime}\right) \leq \phi, d d^{\prime} \leq x^{1 / 4},\left(d, d^{\prime}\right)=1\right\} .
\end{aligned}
$$

If $\left(d, d^{\prime}\right) \in \mathcal{E}$, then clearly the condition $n \in \mathcal{N}_{0}$ in the last factor in (3.8) can be replaced by $n \leq x$ so that, by (3.15), (3.8) can be rewritten as

$$
S=\sum_{\left(d, d^{\prime}\right) \in \mathcal{E}} f\left(d d^{\prime}\right)\left|\left\{n: n \leq x, d(n)=d, d(n+1)=d^{\prime}\right\}\right| .
$$

For fixed $\left(d, d^{\prime}\right) \in \mathcal{E}, n$ satisfies (3.10) if and only if representation (2.1) of $n$ and $n+1$ is of the form

$$
\begin{aligned}
n & =d m, \quad & \left(m, P_{y}\right) & =1, \\
n+1 & =d^{\prime} m^{\prime}, \quad & \left(m^{\prime}, P_{y}\right) & =1 .
\end{aligned}
$$

By (3.15), there are uniquely defined integers $a, a^{\prime}$ with

$$
a^{\prime} d^{\prime}-a d=1, \quad-d^{\prime}<a \leq 0 .
$$

Then the positive integers $m, m^{\prime}$ satisfy

$$
(n=) d m=d^{\prime} m^{\prime}-1 \leq x
$$

if and only if there is a positive integer $r$ with

$$
m=d^{\prime} r+a, \quad m^{\prime}=d r+a^{\prime}, \quad r \leq(x-a d) /\left(d d^{\prime}\right) .
$$

By (3.17), (3.18) and (3.20), we may rewrite (3.16) as

$$
\begin{aligned}
S=\sum_{\left(d, d^{\prime}\right) \in \mathcal{E}} f\left(d d^{\prime}\right) \mid\left\{r: r \leq(x-a d) /\left(d d^{\prime}\right),\right. \\
\left.\left(\left(d^{\prime} r+a\right)\left(d r+a^{\prime}\right), P_{y}\right)=1\right\} \mid .
\end{aligned}
$$

If $\left(d, d^{\prime}\right) \in \mathcal{E}$, then by the definition of $\mathcal{E}$ we have

$$
d d^{\prime} \leq x^{1 / 4} .
$$


It follows from (3.19) and (3.22) that

$$
\left(\frac{x}{d d^{\prime}}+1 \geq\right) \frac{x-a d}{d d^{\prime}} \geq \frac{x}{d d^{\prime}} \geq x^{3 / 4} .
$$

If $x^{3 / 4} \leq y \leq x$, then (1.8) holds trivially; thus we may assume that $y<x^{3 / 4}$. Then by (3.23), Lemma 1 can be applied with $(x-a d) /\left(d d^{\prime}\right)$ and $\left(d^{\prime} n+a\right)$ $\times\left(d n+a^{\prime}\right)$ in place of $x$ and $F(n)$, respectively. (Note that $\left(a_{1}, b_{1}\right)=\left(d^{\prime}, a\right)=1$ and $\left(a_{2}, b_{2}\right)=\left(d, a^{\prime}\right)=1$ follow from (3.19) and $\left(a_{1}, a_{2}\right)=\left(d^{\prime}, d\right)=1$ also holds by (3.15).)

Then, by (3.19),

$$
E=a_{1} a_{2}\left(a_{1} b_{2}-a_{2} b_{1}\right)=d d^{\prime}\left(d^{\prime} a^{\prime}-d a\right)=d d^{\prime},
$$

clearly,

$$
\varrho(p)=1 \quad \text { for } p \mid E,
$$

and, by (3.23),

$$
v=\frac{\log (x-a d) /\left(d d^{\prime}\right)}{\log y} \geq \frac{3}{4} \cdot \frac{\log x}{\log y}=\frac{3}{4} u .
$$

Thus we deduce from Lemma 1 that for $\left(d, d^{\prime}\right) \in \mathcal{E}$ we have

$$
\begin{aligned}
\mid\{r: r \leq & \left.(x-a d) /\left(d d^{\prime}\right),\left(\left(d^{\prime} r+a\right)\left(d r+a^{\prime}\right), P_{y}\right)=1\right\} \mid \\
= & \frac{x-a d}{d d^{\prime}} \prod_{p \mid d d^{\prime}}\left(1-\frac{1}{p}\right) \prod_{\substack{p \nmid d d^{\prime} \\
p \leq y}}\left(1-\frac{2}{p}\right) \\
& \times\left(1+O\left(\exp \left(-\frac{u}{2} \log u\right)+\exp (-\sqrt{\log x})\right)\right) \\
= & \frac{x}{d d^{\prime}} \prod_{p \mid d d^{\prime}}\left(1-\frac{1}{p}\right) \prod_{\substack{p \nmid d d^{\prime} \\
p \leq y}}\left(1-\frac{2}{p}\right) \\
& \times\left(1+O\left(\exp \left(-\frac{u}{2} \log u\right)+\exp (-\sqrt{\log x})\right)\right)+O(1) .
\end{aligned}
$$

Observe that this estimate depends only on the value of the product $d d^{\prime}$ but it is independent of $a, a^{\prime}$ and the factors $d, d^{\prime}$. Thus introducing the notation

$$
D=d d^{\prime},
$$

we infer from (3.21) and (3.24) that 


$$
\begin{aligned}
S= & \sum_{D}\left|\left\{\left(d, d^{\prime}\right):\left(d, d^{\prime}\right) \in \mathcal{E}, d d^{\prime}=D\right\}\right| \\
& \times f(D)\left(\frac{x}{D} \prod_{p \mid D}\left(1-\frac{1}{p}\right) \prod_{\substack{p \nmid D \\
p \leq y}}\left(1-\frac{2}{p}\right)\right. \\
& \left.\times\left(1+O\left(\exp \left(-\frac{u}{2} \log u\right)+\exp (-\sqrt{\log x})\right)\right)+O(1)\right)
\end{aligned}
$$

where $D$ runs over all integers that can be represented in the form (3.25) with $\left(d, d^{\prime}\right) \in \mathcal{E}$; denote the set of these integers $D$ by $\mathcal{D}$. If $D \in \mathcal{D}$, then by (3.11)-(3.14) we have

$$
\begin{aligned}
D & \in Q_{y}, \\
\omega(D) & \leq \phi, \\
\Omega(D)-\omega(D) & \leq \phi, \\
D & \leq x^{1 / 4} .
\end{aligned}
$$

Conversely, if $D$ satisfies (3.27)-(3.30), then clearly for any pair $\left(d, d^{\prime}\right)$ satisfying (3.15) and (3.25) we have $\left(d, d^{\prime}\right) \in \mathcal{E}$.

It follows that

$$
\mathcal{D}=\left\{D: D \in Q_{y}, \omega(D) \leq \phi, \Omega(D)-\omega(D) \leq \phi, D \leq x^{1 / 4}\right\}
$$

and clearly

$$
\begin{aligned}
\mid\left\{\left(d, d^{\prime}\right):\left(d, d^{\prime}\right) \in \mathcal{E},\right. & \left.d d^{\prime}=D\right\} \mid \\
& =\left|\left\{\left(d, d^{\prime}\right): d d^{\prime}=D,\left(d, d^{\prime}\right)=1\right\}\right|=2^{\omega(D)} .
\end{aligned}
$$

Moreover, it follows from (3.30) that in (3.26) the last error term $O(1)$ is much smaller than the other error terms, thus it can be dropped. Hence, by (1.6), it follows from (3.26) and (3.32) that

$$
\begin{aligned}
S= & x \sum_{D \in \mathcal{D}} \frac{f(D) 2^{\omega(D)}}{D} \prod_{p \mid D}\left(1-\frac{1}{p}\right) \prod_{\substack{p \nmid D \\
p \leq y}}\left(1-\frac{2}{p}\right) \\
& +O\left(x \exp \left(-\frac{u}{2} \log u\right) \sum_{D \in \mathcal{D}} \frac{2^{\omega(D)}}{D} \prod_{p \mid D}\left(1-\frac{1}{p}\right) \prod_{\substack{p \nmid D \\
p \leq y}}\left(1-\frac{2}{p}\right)\right) \\
& +O\left(x \exp (-\sqrt{\log x}) \sum_{D \in \mathcal{D}} \frac{2^{\omega(D)}}{D} \prod_{p \mid D}\left(1-\frac{1}{p}\right) \prod_{\substack{p \nmid D \\
p \leq y}}\left(1-\frac{2}{p}\right)\right) .
\end{aligned}
$$


Now, as in [4], we define the multiplicative arithmetic function $\psi(n)$ with

$$
\psi\left(p^{\alpha}\right)= \begin{cases}\left(1-\frac{1}{p}\right)\left(1-\frac{2}{p}\right)^{-1}=\frac{p-1}{p-2} & \text { for } p>2, \quad \alpha \in \mathbb{N}, \\ 1 & \text { for } p=2,\end{cases}
$$

and we write

$$
\eta(D)= \begin{cases}1 / 2 & \text { for } 2 \mid D, \\ 0 & \text { for } 2 \nmid D,\end{cases}
$$

so that

$$
\left|\psi\left(p^{\alpha}\right)\right| \leq 2 \quad \text { for all } p \text { and } \alpha \in \mathbb{N} ;
$$

a little computation shows that also

$$
\begin{aligned}
\left|\psi\left(p^{\alpha}\right)\right| & =\left(1-\frac{1}{p}\right)\left(1-\frac{2}{p}\right)^{-1} \leq 1+\frac{1}{p}+\frac{6}{p^{2}} \\
& <\left(1+\frac{1}{p}\right)\left(1+\frac{6}{p^{2}}\right) \leq\left(1+\frac{1}{p}\right) \exp \left(\frac{6}{p^{2}}\right),
\end{aligned}
$$

and

$$
|\eta(D)| \leq 1 \quad \text { for all } D \in \mathbb{N} .
$$

Then the first sum, say $\sum_{1}$, in (3.33) can be rewritten as

$$
\begin{aligned}
\sum_{1} & =\sum_{D \in \mathcal{D}} \frac{f(D) 2^{\omega(D)}}{D} \prod_{p \mid D}\left(1-\frac{1}{p}\right) \prod_{\substack{p \nmid D \\
p \leq y}}\left(1-\frac{2}{p}\right) \\
& =\prod_{2<p \leq y}\left(1-\frac{2}{p}\right) \sum_{D \in \mathcal{D}} \frac{f(D) 2^{\omega(D)} \eta(D) \psi(D)}{D} .
\end{aligned}
$$

Now write

$$
\begin{aligned}
& \mathcal{D}_{1}=\left\{D: D \in Q_{y}, \omega(D)>\phi\right\}, \\
& \mathcal{D}_{2}=\left\{D: D \in Q_{y}, \Omega(D)-\omega(D)>\phi\right\}, \\
& \mathcal{D}_{3}=\left\{D: D \in Q_{y}, D>x^{1 / 4}\right\},
\end{aligned}
$$

so that

$$
\mathcal{D}=Q_{y} \backslash\left(\mathcal{D}_{1} \cup \mathcal{D}_{2} \cup \mathcal{D}_{3}\right) .
$$

It follows from (1.6) and (3.34)-(3.38) that

$$
\begin{aligned}
\sum_{1}= & \prod_{2<p \leq y}\left(1-\frac{2}{p}\right)\left(\sum_{D \in Q_{y}} \frac{f(D) 2^{\omega(D)} \eta(D) \psi(D)}{D}\right. \\
& \left.+O\left(\sum_{D \in \mathcal{D}_{1} \cup \mathcal{D}_{2} \cup \mathcal{D}_{3}} \frac{|f(d)| 2^{\omega(D)} \eta(D) \psi(D)}{D}\right)\right)
\end{aligned}
$$




$$
\begin{aligned}
= & \prod_{2<p \leq y}\left(1-\frac{2}{p}\right) \sum_{D \in Q_{y}} \frac{f(D) 2^{\omega(D)} \eta(D) \psi(D)}{D} \\
& +O\left(( \operatorname { l o g } y ) ^ { - 2 } \left(\sum_{D \in \mathcal{D}_{1}} \frac{2^{\omega(D)} 2^{\omega(D)}}{D}\right.\right. \\
& \left.\left.+\sum_{D \in \mathcal{D}_{2}} \frac{2^{\omega(D)} 2^{\omega(D)}}{D}+\sum_{D \in \mathcal{D}_{3}} \frac{2^{\omega(D)} \psi(D)}{D}\right)\right) \\
= & \prod_{2<p \leq y}\left(1-\frac{2}{p}\right) \sum_{D \in Q_{y}} \frac{f(D) 2^{\omega(D)} \eta(D) \psi(D)}{D} \\
& +O\left(( \operatorname { l o g } y ) ^ { - 2 } \left(\sum_{D \in Q_{y}} \frac{4^{\omega(D)}}{D}\right.\right. \\
& \left.\left.+\sum_{\substack{\omega(D)>\phi \\
\sum^{\omega(D)}}}+\sum_{D \in Q_{y}} \frac{2^{\omega(D)} \psi(D)}{D}\right)\right) \\
= & +O\left((\log y)^{-2}\left(R_{1}+R_{2}+R_{3}\right)\right), \quad \text { say. }
\end{aligned}
$$

Here we have

$$
\begin{aligned}
& Z=\prod_{2<p \leq y}\left(1-\frac{2}{p}\right) \sum_{\alpha=1}^{\infty} \sum_{\substack{D^{\prime} \in Q_{y} \\
2 \nmid D^{\prime}}} \frac{f\left(2^{\alpha}\right) f\left(D^{\prime}\right) 2^{\omega\left(D^{\prime}\right)+1} \psi\left(D^{\prime}\right)}{2^{\alpha} D^{\prime}} \cdot \frac{1}{2} \\
& =\prod_{2<p \leq y}\left(1-\frac{2}{p}\right) \sum_{\substack{D^{\prime} \in Q_{y} \\
2 \nmid D^{\prime}}} \frac{f\left(D^{\prime}\right) 2^{\omega\left(D^{\prime}\right)} \psi\left(D^{\prime}\right)}{D^{\prime}} \sum_{\alpha=1}^{\infty} \frac{f\left(2^{\alpha}\right)}{2^{\alpha}} \\
& =\sum_{\alpha=1}^{\infty} \frac{f\left(2^{\alpha}\right)}{2^{\alpha}} \prod_{2<p \leq y}\left(1-\frac{2}{p}\right)\left(1+2 \psi(p) \sum_{\beta=1}^{\infty} \frac{f\left(p^{\beta}\right)}{p^{\beta}}\right)=\prod_{p \leq y} \delta(p)
\end{aligned}
$$

with the function $\delta(p)$ defined by (1.9). If $f(n)$ is completely multiplicative then clearly $\delta(p)$ in (1.9) is, indeed,

$$
\delta(2)=\sum_{\alpha=1}^{\infty}\left(\frac{f(2)}{2}\right)^{\alpha}=\frac{f(2)}{2-f(2)}=\frac{2+f(2)-2}{2-f(2)}
$$

and

$$
\delta(p)=\left(1-\frac{2}{p}\right)\left(1+2 \frac{p-1}{p-2} \cdot \frac{f(p)}{p-f(p)}\right)=\frac{p+f(p)-2}{p-f(p)} \quad \text { for } p>2
$$

as claimed in (1.10). 
The error terms $R_{1}, R_{2}$ can be estimated in the same way as in [4], and these estimates are similar to those of $\left|N_{1}\right|$ and $\left|N_{2}\right|$ earlier:

$$
\begin{aligned}
R_{1} & =\sum_{k>\phi} \sum_{\substack{D \in Q_{y} \\
\omega(D)=k}} \frac{4^{k}}{D} \\
& \leq \sum_{k>\phi} \frac{1}{k !}\left(\sum_{p \leq y} \sum_{\alpha=1}^{\infty} \frac{4}{p^{\alpha}}\right)^{k}=\sum_{k>\phi} \frac{1}{k !}\left(4 \sum_{p \leq y} \frac{1}{p-1}\right)^{k} \leq \sum_{k>\phi} \frac{1}{k !}\left(8 B_{y}\right)^{k}
\end{aligned}
$$

whence, by

$$
B_{y}=\sum_{p \leq y} \frac{1}{p}=\log \log y+O(1)
$$

and Stirling's formula,

$$
R_{1} \ll \sum_{k>\phi}\left(\frac{24 B_{y}}{k}\right)^{k}<\sum_{k>\phi} 2^{-k} \ll 2^{-\phi}=O\left((\log y)^{-30}\right) .
$$

To estimate $R_{2}$, as in [4] we write each $D$ with $D \in Q_{y}, \Omega(D)-\omega(D)>\phi$ in the form $D=r^{2} s$ with $|\mu(s)|=1$. Then as in the proof of (4.11) in [4] we have $\Omega(r)>\phi / 2$, whence, by (3.41),

$$
r^{2} \geq\left(2^{\Omega(r)}\right)^{2} \geq 2^{\phi}=2^{60 B_{y}}>(\log y)^{36} .
$$

It follows that

$$
\begin{aligned}
R_{2} & =\sum_{\substack{D \in Q_{y} \\
\Omega(D)-\omega(D)>\phi}} \frac{4^{\omega(D)}}{D} \\
& \leq \sum_{r^{2}>(\log y)^{36}} \sum_{\substack{s \in Q_{y} \\
|\mu(s)|=1}} \frac{4^{\omega\left(r^{2} s\right)}}{r^{2} s} \\
& \leq \sum_{r^{2}>(\log y)^{36}} \frac{4^{\omega\left(r^{2}\right)}}{r^{2}} \sum_{\substack{s \in Q_{y} \\
|\mu(s)|=1}} \frac{4^{\omega(s)}}{s} \\
& \ll(\log y)^{-17} \prod_{p \leq y}\left(1+\frac{4}{p}\right) \leq(\log y)^{-17} \exp \left(4 B_{y}\right) \\
& \ll(\log y)^{-17}(\log y)^{4}=(\log y)^{-13} .
\end{aligned}
$$

To estimate $R_{3}$, observe that by (3.35) we have

$$
|\psi(D)|=\prod_{p^{\alpha} \| D}\left|\psi\left(p^{\alpha}\right)\right|<\prod_{p \mid D}\left(1+\frac{1}{p}\right) \exp \left(\sum_{p} \frac{6}{p^{2}}\right) \ll \prod_{p \mid D}\left(1+\frac{1}{p}\right)
$$


so that

$$
R_{3} \ll \sum_{\substack{D \in Q_{y} \\ D>x^{1 / 4}}} \frac{1}{D} 2^{\omega(D)} \prod_{p \mid D}\left(1+\frac{1}{p}\right)
$$

The function

$$
h(D)=2^{\omega(D)} \prod_{p \mid D}\left(1+\frac{1}{p}\right)
$$

is multiplicative, we have

$$
h(p)=h\left(p^{2}\right)=\ldots=h\left(p^{\alpha}\right)=\ldots=2\left(1+\frac{1}{p}\right)
$$

so that (2.3) holds, and it also satisfies (2.4) with $K=2$ and $L=2$. Thus the sum in (3.44) can be estimated by using Lemma 3 with $x^{1 / 4}$ in place of $z$. We obtain

$$
R_{3} \ll(\log y)^{2} \exp \left(-\frac{\log x^{1 / 4}}{\log y}\right)=(\log y)^{2} \exp \left(-\frac{u}{4}\right) .
$$

Finally, by (3.35) and (3.36), the sum in the error term in (3.33) can be estimated in the following way:

$$
\begin{aligned}
\sum_{D \in \mathcal{D}} \frac{2^{\omega(D)}}{D} \prod_{p \mid D}\left(1-\frac{1}{p}\right) \prod_{\substack{p \nmid D \\
p \leq y}}\left(1-\frac{2}{p}\right) \\
=\prod_{2<p \leq y}\left(1-\frac{2}{p}\right) \sum_{D \in \mathcal{D}} \frac{2^{\omega(D)} \eta(D) \psi(D)}{D} \\
\ll(\log y)^{-2} \sum_{D \in \mathcal{D}} \frac{2^{\omega(D)}}{D} \prod_{p \mid D}\left(1+\frac{1}{p}\right) \exp \left(\sum_{p \mid D} \frac{6}{p^{2}}\right) \\
\ll(\log y)^{-2} \sum_{D \in Q_{y}} \frac{2^{\omega(D)}}{D} \prod_{p \mid D}\left(1+\frac{1}{p}\right) \\
=(\log y)^{-2} \prod_{p \leq y}\left(1+\frac{2(p+1)}{(p-1) p}\right) \\
=(\log y)^{-2} \prod_{p \leq y}\left(1+\frac{2}{p}\right) \exp \left(O\left(\frac{1}{p^{2}}\right)\right) \\
\ll(\log y)^{-2} \prod_{p \leq y}\left(1+\frac{2}{p}\right)=O(1) .
\end{aligned}
$$

It follows from (3.33), (3.37), (3.39), (3.40), (3.42), (3.43), (3.45) and (3.46) that 


$$
\begin{aligned}
S= & x \prod_{p \leq y} \delta(p) \\
& +O\left(x(\log y)^{-2}\left((\log y)^{-30}+(\log y)^{-13}+(\log y)^{2} \exp \left(-\frac{u}{4}\right)\right)\right) \\
& +O\left(x\left(\exp \left(-\frac{u}{2} \log u\right)+\exp (-\sqrt{\log x})\right)\right) \\
= & x \prod_{p \leq y} \delta(p)+O\left(x\left((\log y)^{-15}+\exp \left(-\frac{u}{4}\right)+\exp (-\sqrt{\log x})\right)\right) .
\end{aligned}
$$

It is easy to see that here we have

$$
\exp (-\sqrt{\log x}) \ll \max \left\{(\log y)^{-15}, \exp \left(-\frac{u}{4}\right)\right\},
$$

thus the $\exp (-\sqrt{\log x})$ term in the error term can be dropped:

$$
S=x \prod_{p \leq y} \delta(p)+O\left(x\left((\log y)^{-15}+\exp \left(-\frac{u}{4}\right)\right)\right) .
$$

(1.8) in the Theorem follows from (3.1)-(3.6) and (3.47) and this completes the proof of the Theorem.

\section{References}

[1] N. G. de Bruijn, On the number of positive integers $\leq x$ and free of prime factors $>y$, Nederl. Akad. Wetensch. Proc. Ser. A 54 (1951), 50-60; Part II, 69 (1966), 239-247.

[2] E. R. Canfield, P. Erdős and C. Pomerance, On a problem of Oppenheim concerning "Factorisatio Numerorum", J. Number Theory 17 (1983), 1-28.

[3] J. Cassaigne, S. Ferenczi, C. Mauduit, J. Rivat and A. Sárközy, On finite pseudorandom binary sequences III: The Liouville function, I, Acta Arith. 87 (1999), 367-390.

[4] H. Daboussi and A. Sárközy, On pseudorandom properties of multiplicative functions, Acta Math. Acad. Sci. Hungar., to appear.

[5] H. Halberstam and H.-E. Richert, Sieve Methods, Academic Press, London, 1974.

[6] A. Hildebrand, Math. Rev., review no. 95d:11099.

Faculté de Mathématiques et d'Informatique

Université de Picardie

33 rue Saint Leu

80039 Amiens Cedex 01, France

E-mail: Hedi.Daboussi@u-picardie.fr
Department of Algebra and Number Theory Eötvös Loránd University Pázmány Péter sétány 1/C 1117 Budapest, Hungary E-mail: sarkozy@cs.elte.hu 